\title{
Applications of MIMO Techniques to Sensing of Cardiopulmonary Activity
}

\author{
Dragan Samardzija ${ }^{1}$, Olga Boric-Lubecke ${ }^{2}$, Anders Host-Madsen ${ }^{2}$, Victor M. Lubecke ${ }^{2}$, \\ Theodore Sizer, $\mathrm{II}^{1}$, Amy D. Droitcour ${ }^{3}$, and Gregory T. A. Kovacs ${ }^{3}$ \\ ${ }^{1}$ Bell Laboratories, Lucent Technologies, L-273, 791 Holmdel-Keyport Road \\ Holmdel, NJ 07733, USA, dragan@bell-labs.com \\ ${ }^{2}$ Department of Electrical Engineering, University of Hawaii at Manoa, 2540 Dole St, \\ Honolulu, HI 96822, USA, olga@ieee.org \\ ${ }^{3}$ Department of Electrical Engineering, Stanford University, Stanford, CA 94305, USA
}

Abstract- Remote monitoring of cardiopulmonary activity based on Doppler shifts in radio signals shows promise in medical and security applications, however the problems of motion artifacts and presence of multiple subjects limit the usefulness of this technique. By applying MIMO signal processing, it is possible to overcome limitations of current systems and isolate signals from multiple sources.

\section{Introduction}

Remote sensing of heart and respiration activity by measuring Doppler shift in radio signals is a promising technique for unobtrusive health monitoring and life sensing, with proof of concept demonstrated for various applications [1-3]. Unfortunately, this principle has not been developed to the level of practical applications, mainly due to a lack of effective way to isolate desired target motion from other motion and other targets. We are exploring an approach to isolate desired scattered signals from unwanted motion based on recent developments in multiple input multiple output (MIMO) signal processing techniques. Lucent Technologies has been the leader in this field with BellLabs LAyered Space-Time (BLAST) techniques [4]. We propose to use techniques based on BLAST to isolate useful scattered radio signals from those due to unwanted motion in the environment. If there is interfering motion in the radar field of view, multiple antennas can be used to detect multiple copies of the same signal with different phase information, with coherent combining used to provide a greatly improved estimate of desired Doppler motion. When more than one target is in view, multiple transmitters and receivers providing multiple signal copies could be used to distinguish between the different sources of Doppler motion, isolate the desired signal, and determine a number of targets. In this paper, we present MIMO system simulation results that indicate that it is possible to separate multiple subjects even with identical breathing and heart rates.

\section{MIMO System Model and Numerical Results}

We will consider a MIMO system consisting of $n_{t x}$ transmit and $n_{r x}$ receive antennas, forming two proper linear antenna arrays. The arrays are spatially separated, for example, occupying two opposite walls in a room while facing each other (Fig. 1(a)). Using the well-established model, the MIMO channel is represented as an $n_{r x} \times n_{t x}$ matrix $\mathbf{H}$ where $h_{i j}$ is its $i t h$ row and $j t h$ column element, and $h_{i j}$ corresponds to the channel response (i.e., channel state) between the $i t h$ receive and $j$ th transmit antenna $\left(i=1, \ldots, n_{r x}\right.$ and $j=1, \ldots$, $n_{t x}$ ). Each transmit antenna is emitting a unique pilot signal. For example, the pilot signals are orthogonal symbol sequences where each transmit antenna emits one sequence and it is orthogonal to the ones assigned to the other transmit antennas (e.g., a CDMA-like signaling). The MIMO receiver has knowledge of the pilot signals, and it applies channel estimation to learn the channel response between each transmit and receive antenna. Consequently, an estimate of the channel $h_{i j}$ is $\underline{h}_{i j}=h_{i j}+n_{i j}$, where $n_{i j}$ is the additive 
white Gaussian noise (AWGN) corresponding to the thermal noise. The noise has a zeromean complex Gaussian distribution with the variance $N_{e}$ given as

$$
\left.N_{e}\right|_{d B m}=10 \log _{10}(K T B)-G_{e}+30[\mathrm{dBm}]
$$

where $K$ is Boltzmann's constant, $T$ is the environment temperature, $B$ is the signal bandwidth and $G_{e}$ is a gain introduced by the estimation procedure (by averaging over a number of symbol periods, where the symbol period is $T_{\text {sym }}=1 / B$ ). We assume that a person whose cardiopulmonary activity is being observed is positioned somewhere between the transmit and receive antenna arrays (Fig. 1 (a)). To numerically assess the performance of the MIMO radar sensing system, we model a human body as a cluster of isotropic scatterers. The scatterers are randomly distributed around the cluster center, within a circle of diameter $D$. The cluster contains $N_{s}$ scatterers.

Simulations were done for a MIMO system with 4 transmit and 4 receive antennas, with the carrier frequency of $2.4 \mathrm{GHz}$, and $2 \lambda$ and $\lambda / 2$ separation between transmit and receive antenna elements, respectively (where $\lambda$ is the wavelength). The two arrays are 4 meters apart. The scatterer cluster of 10 scatterers is randomly positioned, with the diameter $D=0.5 \mathrm{~m}$. Note that the effective area $S_{s}$ of each isotropic scatterer is selected with the assumption that half of the incident energy on the human body is reflected. We assume that the area of the human body, orthogonal to the incident electromagnetic wave, is $S_{h}$ (we use $S_{h}=1.7 \times 0.3 \mathrm{~m}^{2}$ ) Consequently, the effective area of each isotropic scatterer is $S_{s}=S_{h} /\left(2 N_{s}\right)$. Based on the above assumptions, the channel response between the $i t h$ receive and $j$ th transmit antenna $\left(i=1, \ldots, n_{r x}\right.$ and $\left.j=1, \ldots, n_{t x}\right)$ is

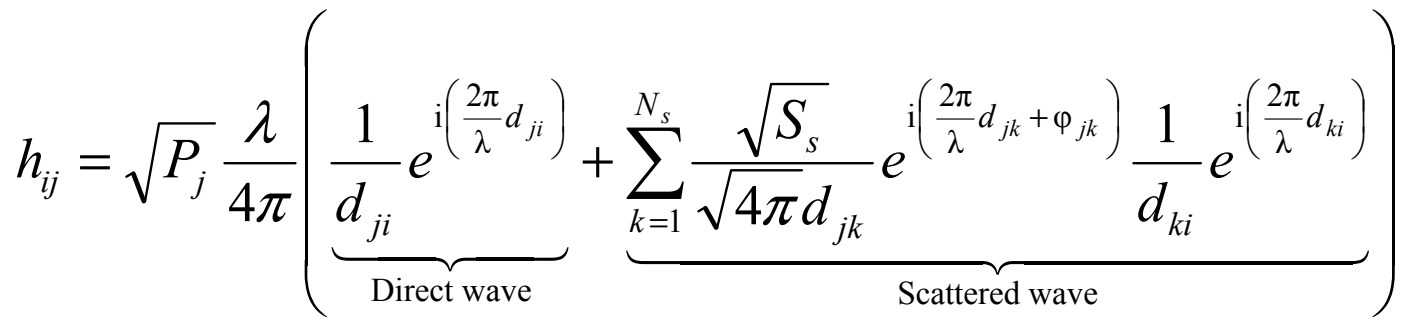

where $d_{j k}$ the distance between transmit antenna $j$ and scatterer $k, d_{k i}$ is the distance between scatterer $k$ and receive antenna $i, d_{j i}$ is the distance between transmit antenna $j$ and receive antenna $i$, and finally, $\varphi_{j k}$ is a random phase shift. In the above derivations, receive antennas are assumed to be isotropic with $0 \mathrm{~dB}$ gain. To model the cardiopulmonary activity the position of each scatterer, with respect to the cluster center, is oscillating in time on two different frequencies with different amplitudes. The first frequency and amplitude model the cardio activity (typical frequency is $1 \mathrm{~Hz}$ and the amplitude is $0.1 \mathrm{~mm}$ ), while the second frequency and amplitude model the pulmonary activity (typical frequency is $0.3 \mathrm{~Hz}$ and the amplitude is $10 \mathrm{~mm}$ ) [5,6]. To analyze temporal variations in the MIMO channel, we collect a sequence of the channel state estimates $\underline{h}_{i j}(k)\left(i=1, \ldots, n_{r x}\right.$ and $\left.j=1, \ldots, n_{t x}\right)$ where $k$ is the temporal index. The total of $n_{r x} \times n_{t x}$ sequences is available (each sequence corresponding to one transmitterreceiver pair).

Figure 1 (b) shows the power spectrum of the channel state estimate sequence averaged over all transmitter-receiver pairs. The accumulative Tx power from all Tx antennas is 20 $\mathrm{dBm}$, while the signal bandwidth is $1 \mathrm{MHz}$, and estimation gain is $20 \mathrm{~dB}$ (which corresponds to integration over 100 pilot symbols, i.e., $100 \mu \mathrm{sec}$ ). The MIMO channel state sampling frequency is $10 \mathrm{~Hz}$ and the total of 100 samples/Rx-Tx pair is collected. 


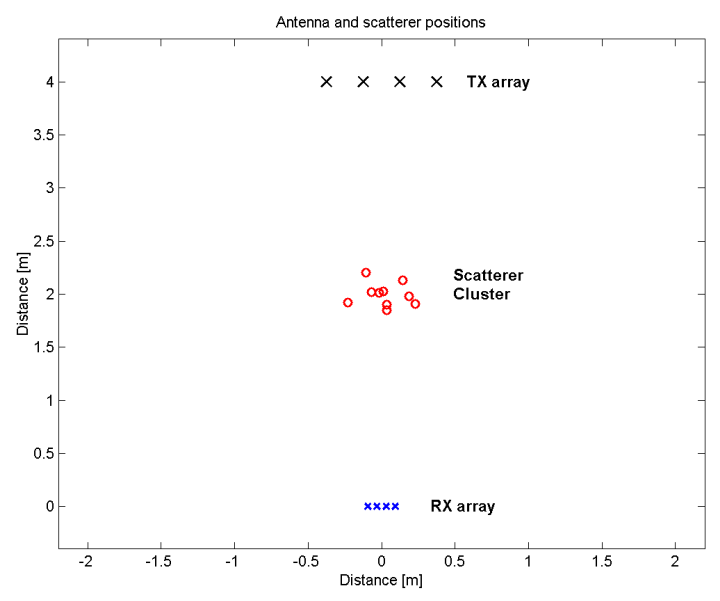

(a)

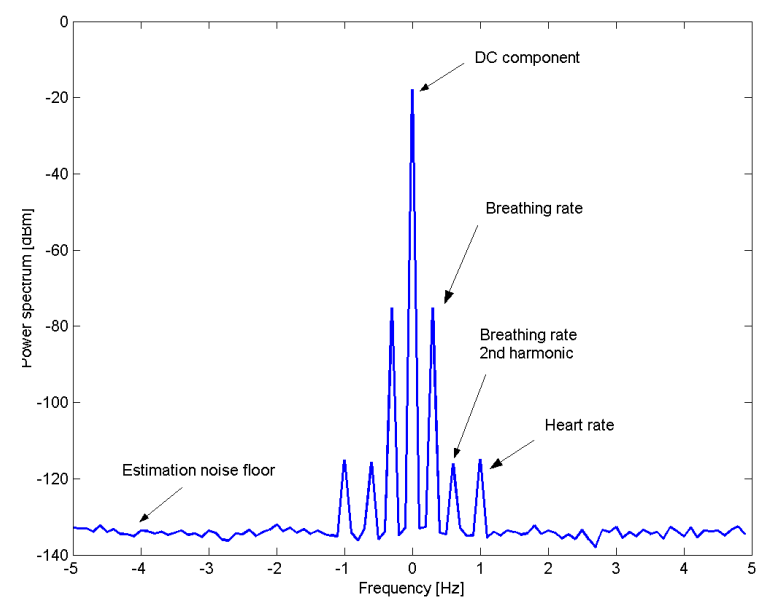

(b)

Fig.1 A typical arrangement of Tx and Rx antennas, and cluster of scatterers that models a human body (a), and the power spectrum of the channel state estimate sequence (b).

We note that the sensing of the cardio activity is far more challenging and adversely affected by the noise in the system. The pulmonary signal is significantly stronger, however its frequency is closer to the $60 \mathrm{~dB}$ higher DC component. In light of the above results, the receiver radio-frequency front-end and analog-to-digital converters have to have low noise figure, low phase noise and large linear dynamic range (e.g., in this particular example analog-to-digital converters with 20 or more bits would be required).

To illustrate potentials of the multiple antenna systems to exploit certain spatial aspects, we observe a case of two individuals with same frequency and amplitude of the cardiopulmonary activity at different locations in the room that are not a priori known to the receiver. After performing the temporal spectrum analysis, similar results to the ones presented in Fig. 1 (b) are obtained, and it is not possible to distinguish multiple subjects. In order to estimate spatial characteristics of specific spectral components of the channel state estimate sequence, we perform the following steps: (1) Determine the power spectrum of the channel state estimate sequence averaged over all Tx-Rx pairs (i.e., like the results in Figure 1(b)); (2) Select a frequency component of interest (e.g., component denoted as the breathing rate in Figure 1(b)). For individual Tx-Rx pair (there is total of $n_{r x} \times n_{t x}$ pairs), perform the Fourier transformation and select the coefficient corresponding to the desired frequency component. We denote the selected coefficient as $c_{i j}\left(i=1, \ldots, n_{r x}\right.$ and $\left.j=1, \ldots, n_{t x}\right)$ corresponding to the $i$ th $\mathrm{Rx}$ and $j$ th $\mathrm{Tx}$ antenna. Arrange the coefficients in a matrix $\mathbf{C}$ (where $c_{i j}$ occupies $i$ th row and $j$ th column); (3) Define a vector $r(\alpha)$ where $d$ is the distance between the $\mathrm{Rx}$ antenna elements

(set to $\lambda / 2)$ :

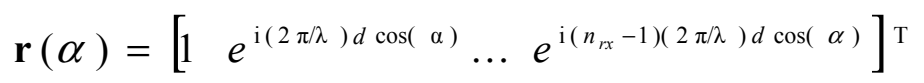


(4) Determine how much power is arriving to the Rx antenna array from the direction corresponding to angle $\alpha$ by performing the projection operation, $P_{a r}(\alpha)=\left|\mathrm{C}^{\mathrm{H}} \mathrm{r}(\alpha)\right|^{2}$, where $P_{a r}(\alpha)$ is denoted as the angular power density. Figure 2(b) presents the normalized angular power density for the individuals located as given in Figure 2 (a). The results are presented for the different number of Rx antennas $\left(n_{r x}=4,8\right.$ and 16). We can clearly see two dominant directions of arrival at angles $45^{\circ}$ and $135^{\circ}$ (corresponding to the cluster 1 and 2 , respectively). We also note that as the number of antenna increases the maximums are becoming narrower allowing for more accurate estimation of the direction of arrival. Similar results can be obtained if the number of Tx antennas is varied.

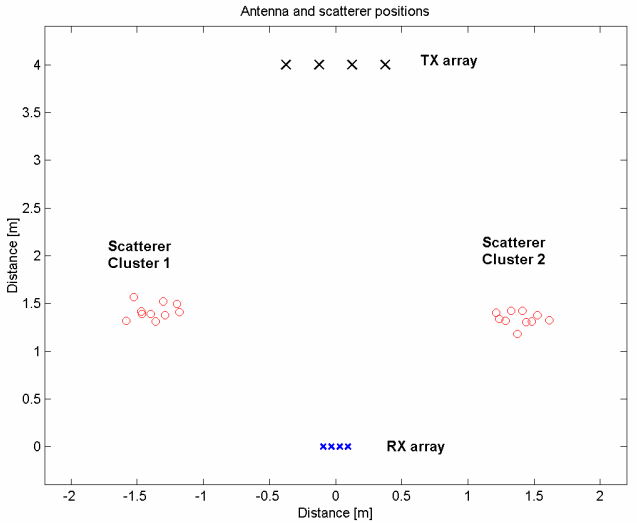

(a)

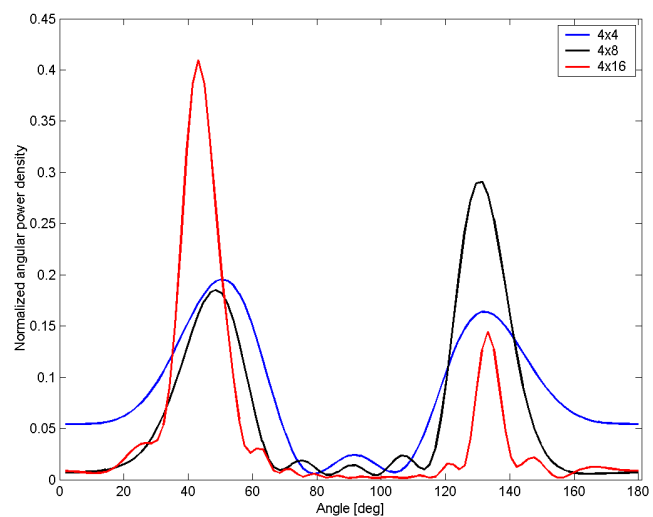

(b)

Fig. 2 An arrangement of Tx and Rx antennas, and two clusters of scatterers (a), and the normalized angular power density for different number of Rx antennas and two individuals (b).

\section{Conclusions}

In this paper we explored the application of MIMO systems to detection of cardiopulmonary signals resulting from Doppler shifts in radio signals. In future work the effect of different number of antennas and antenna arrangements on system performance will be investigated

\section{References}

[1] J. C. Lin, "Microwave sensing of physiological movement and volume change: a review," Bioelectromagnetics, vol. 13, pp. 557-565, 1992.

[2] K. M. Chen, D. Mirsa, H. Wang, H. R. Chuang, E. Postow, "An X-band microwave life detection system," IEEE Transactions of Biomedical Eng., vol. 33, pp. 697-70, 1986.

[3] V.M. Lubecke, O. Boric-Lubecke, G. Awater, P.-W. Ong, P. Gammel, R.-H. Yan, J.C. Lin, "Remote sensing of vital signs with telecommunications signals," World Congress on Medical Physics and Biomedical Engineering (WC2000), Chicago IL, July 2000.

[4] G. J. Foschini, "Layered Space-Time Architecture for Wireless Communication in a Fading Environment When Using Multiple Antennas," Bell Labs Technical Journal, Vol. 1, No. 2, Autumn 1996, pp 41-59.

[5] T. Kondo, T. Uhlig, P. Pemberton, P. D. Sly, "Laser Monitoring of Chest Wall Displacement," Eur. Respir. Journal, Vol. 10, pp. 1865-1869, 1997.

[6] G. Ramachandran, S. Swarnamani, and M. Singh, "Reconstruction of Out-of-Plane Cardiac Displacement Patterns as Observed on the Chest Wall During Various Phases of ECG by Capacitance Transducer," IEEE Trans. on Biomed. Eng., Vol. 38, No. 4 pp. 383-385, 1991. 